\title{
Tumor de Wilms Bilateral Sincrônico: Avaliação Cirúrgica e Sobrevida
}

doi: https://doi.org/10.32635/2176-9745.RBC.2018v64n3.33

Synchronous Bilateral Wilms Tumor: Surgical Evaluation and Survival

Tumor de Wilms Bilateral Sincrónico: Evaluación Quirúrgica y Sobrevida

Pablo Baptista Oliveira'; Marilia Fornaciari Grabois²; Fernanda Ferreira da Silva Lima ${ }^{3}$; Paulo Antônio Silvestre de Faria ${ }^{4}$; Vanessa do Nascimento Santos ${ }^{5}$; Francisca Norma Albuquerque Girão Gutierrez ${ }^{6}$; Sima Esther Ferman ${ }^{7}$; Ricardo Vianna de Carvalho ${ }^{8}$

Resumo

Introduçáo: Os tumores renais representam cerca de $7 \%$ de todas as neoplasias malignas da infância. O tumor de Wilms bilateral sincrônico corresponde a 5-7\% de todos os nefroblastomas. O tratamento consiste em quimioterapia pré-operatória, seguida de cirurgia conservadora, quimioterapia pós-operatória e, quando indicada, radioterapia. Objetivo: Analisar o tipo de cirurgia e a sobrevida global dos pacientes com tumor de Wilms bilateral sincrônico. Método: Este estudo de coorte retrospectivo incluiu 18 pacientes pediátricos do Hospital do Câncer I, do Instituto Nacional de Câncer José Alencar Gomes da Silva, no Rio de Janeiro, de janeiro de 2000 a dezembro de 2017. Curvas de sobrevida foram calculadas pelo método Kaplan-Meier. Resultados: A idade mediana ao diagnóstico foi de 19 meses, sendo dez casos do sexo feminino. Em 62,5\% dos rins operados, foi possível realizar cirurgia conservadora e, em 41,2\% (7/17) dos pacientes, em ambos os rins. A sobrevida global em cinco anos, segundo o tipo cirurgia, foi de $87,5 \%$ para cirurgia conservadora e de $62,8 \%$ para nefrectomia total $(\mathrm{p}=0,0001)$. A sobrevida global em cinco anos para a coorte inteira foi de $70,8 \%$. Conclusáo: A cirurgia preservadora de tecido renal em crianças com o tumor de Wilms bilateral sincrônico é viável e pode ser realizada com segurança em centros de referência. Palavras-chave: Tumor de Wilms Bilateral; Criança; Adolescente; Nefrectomia; Análise de Sobrevida.

\section{Abstract}

Introduction: Kidney tumors account for about $7 \%$ of all childhood malignancies. Synchronous bilateral Wilms tumor corresponds to $5-7 \%$ of all nephroblastomas. The treatment consists of preoperative chemotherapy, followed by conservative surgery, postoperative chemotherapy and, when indicated, radiotherapy. Objective: To analyze the type of surgery and the overall survival of patients with synchronous bilateral Wilms tumor. Method: This retrospective cohort study included 18 pediatric patients from the Hospital do Cancer I, from the National Cancer Institute José Alencar Gomes da Silva, in Rio de Janeiro, from January 2000 to december 2017. Survival curves were calculated using the Kaplan-Meier method. Results: The median age at diagnosis was 19 months, and 10 patients were female. It was possible to perform conservative surgery in $62.5 \%$ of the kidneys, and in $41.2 \%$ (7/17) of patients in both kidneys. The 5 -year overall survival according to the type of surgery was $87.5 \%$ for conservative surgery and $62.8 \%$ for radical nephrectomy $(\mathrm{p}=0.0001)$. The 5 -year overall survival of the entire cohort was $70.8 \%$. Conclusion: Conservative surgery in pediatric synchronous bilateral Wilms tumor is feasible and can be performed safely in reference centers.

Key words: Wilms Tumor Bilateral; Child; Adolescent; Nephrectomy; Survival Analysis.
Resumen

Introducción: Los tumores renales representan alrededor del 7\% de todas las neoplasias malignas de la infancia. El tumor de Wilms bilateral sincrónico corresponde al $5-7 \%$ de todos los nefroblastomas. El tratamiento consiste en quimioterapia preoperatoria, seguida de cirugía conservadora, quimioterapia postoperatoria y, cuando indicada, la radioterapia. Objetivo: Analizar el tipo de cirugía y la supervivencia global de los pacientes con un tumor de Wilms bilateral sincrónico. Método: Este estudio de cohorte retrospectivo incluyó 18 pacientes pediátricos del Hospital del Cáncer I, del Instituto Nacional de Cáncer José Alencar Gomes da Silva, nel Río de Janeiro, de enero de 2000 a diciembre de 2017. Las curvas de sobrevida fueron calculadas por el método Kaplan-Meier. Resultados: La edad media al diagnóstico fue de 19 meses, siendo diez casos del sexo femenino. En el 62,5\% de los rińones operados fue posible realizar cirugía conservadora, siendo en el $41,2 \%$ (7/17) de los pacientes en los dos rińones. La supervivencia global en cinco años según el tipo de cirugía fue de $87,5 \%$ para cirugía conservadora y de $62,8 \%$ nefrectomía total $(\mathrm{p}=0,0001)$. La supervivencia global en cinco ańos para la cohorte entera fue del $70,8 \%$. Conclusión: La cirugía conservadora en niños con el tumor de Wilms bilateral sincrónico es viable y puede ser realizada con seguridad en centros de referencia.

Palabras clave: Tumor de Wilms Bilateral; Niño; Adolescente; Nefrectomía; Análisis de Supervivencia.

\footnotetext{
${ }^{1}$ Instituto Nacional de Câncer José Alencar Gomes da Silva (INCA). Rio de Janeiro (RJ), Brasil. Orcid iD: https://orcid.org/0000-0003-4677-8873

${ }^{2}$ INCA. Rio de Janeiro (RJ), Brasil. Orcid iD: https://orcid.org/0000-0002-9368-1030

${ }^{3}$ INCA. Rio de Janeiro (RJ), Brasil. Orcid iD: https://orcid.org/0000-0002-6658-3101

${ }^{4}$ INCA. Rio de Janeiro (RJ), Brasil. Orcid iD: https://orcid.org/0000-0003-1817-4888

${ }^{5}$ INCA. Rio de Janeiro (RJ), Brasil. Orcid iD: https://orcid.org/0000-0003-38434085

${ }^{6}$ INCA. Rio de Janeiro (RJ), Brasil. Orcid iD: https://orcid.org/0000-0002-8107-0136

${ }^{7}$ INCA. Rio de Janeiro (RJ), Brasil. Orcid iD: https://orcid.org/0000-0002-7076-6779

${ }^{8}$ INCA. Rio de Janeiro (RJ), Brasil. Orcid iD: https://orcid.org/0000-0002-3768-8289

Endereço para correspondência: Ricardo Vianna de Carvalho. Hospital do Câncer I. INCA. Praça Cruz Vermelha, 23 - 5 andar (chefia da pediatria) - Centro. Rio de Janeiro (RJ), Brasil. CEP 20230-130. E-mail: cir_oncologica.pediatrica@inca.gov.br.
} 


\section{INTRODUÇÃO}

Os tumores renais representam cerca de $7 \%$ de todas as neoplasias malignas da infância ${ }^{1}$. A taxa de incidência do tumor de Wilms em crianças entre 0-14 anos foi de 8,3 por milhão ${ }^{2}$ nos Estados Unidos da América e de 6,01 por milhão no Brasil ${ }^{1}$. O tumor de Wilms ou nefroblastoma é o mais frequente, correspondendo a $95 \%$ dos casos $^{3}$, podendo acometer um rim (unilateral) ou ambos os rins simultaneamente (bilateral), também denominado de tumor de Wilms bilateral sincrônico.

O tumor de Wilms bilateral sincrônico corresponde a 5-7\% de todos os nefroblastomas ${ }^{4,5}$. Geralmente, ocorre em crianças mais jovens (média de 2,5 anos de idade) em comparaçáo aos tumores unilaterais, além de ser um importante fator de risco para o desenvolvimento de falência renal ${ }^{4}$. Segundo os estudos do National Wilms Tumor Study (NWTS 1-4), 12\% dos pacientes com tumor de Wilms bilateral sincrônico desenvolveram falência renal, principalmente devido à abordagem cirúrgica agressiva ${ }^{6}$.

A terapêutica moderna do tumor de Wilms bilateral sincrônico consiste em quimioterapia pré-operatória, seguida de cirurgia preservadora de tecido renal (CPTR), quimioterapia pós-operatória e, quando indicada, a radioterapia. Nesses pacientes, o tratamento bem-sucedido deve alcançar o controle oncológico, preservando ao máximo o parênquima renal para garantir função renal ${ }^{7}$. A doença renal terminal afeta negativamente a saúde geral e a qualidade de vida do paciente, sendo esses efeitos mais acentuados em crianças pequenas ${ }^{8}$. Crianças com doença renal terminal requerem modificaçóes dietéticas e de estilo de vida, além de terapia substitutiva renal, hospitalizações frequentes e aumento do uso de medicamentos?

O objetivo deste estudo é apresentar as características clínicas e a evolução dos pacientes com tumor de Wilms bilateral sincrônico, com ênfase nos aspectos cirúrgicos, em uma instituição de referência no tratamento do câncer pediátrico. A abordagem desses pacientes deve ser multidisciplinar (cirurgiôes pediátricos, oncologistas pediátricos, patologistas, radiologistas e radioterapeutas). O protocolo adotado para o tratamento desses pacientes foi o padronizado pela Sociedade Internacional de Oncologia Pediátrica (Siop) 2001 e pela Sociedade Brasileira de Oncologia Pediátrica (Sobope).

\section{MÉTODO}

Esta coorte retrospectiva foi composta por todas as crianças com tumor de Wilms bilateral sincrônico, matriculadas no Serviço de Oncologia Pediátrica do Hospital do Câncer I, do Instituto Nacional do Câncer
José Alencar Gomes da Silva (HC I/INCA), no Rio de Janeiro, entre janeiro de 2000 e dezembro de 2017. Foram identificados 259 pacientes com tumor de Wilms, sendo que 18 (7\%) apresentavam exames de imagem sugestivos de tumor renal bilateral. Os critérios de elegibilidade foram: idade até 15 anos, doença renal bilateral sincrônica ao diagnóstico definida pelo estudo de imagem (ultrassonografia, tomografia computadorizada e/ou ressonância magnética) e ausência de doença metastática. Os exames de imagem permitiram identificar a distribuição tumoral multicêntrica em cada rim. Todos os dados utilizados foram coletados a partir de informaçóes registradas em prontuário físico e/ou eletrônico.

As variáveis analisadas foram: idade ao diagnóstico, sexo, apresentação clínica inicial, malformaçôes associadas, quimioterapia pré-operatória, procedimentos cirúrgicos (biópsia, CPTR, nefrectomia parcial e/ou nefrectomia total), histopatologia, estádio cirúrgico, tempo de seguimento e condição clínica ao final da observação. Foram consideradas cirurgias conservadoras a CPTR isolada, a CPTR associada à nefrectomia parcial e a nefrectomia parcial isolada.

Todos os pacientes receberam quimioterapia neoadjuvante, iniciada com duas drogas: dactinomicina $0,045 \mathrm{mg} / \mathrm{kg} / \mathrm{dia}$ (D1 e D15 - total de duas doses) e vincristina $1,5 \mathrm{mg} / \mathrm{m}^{2} /$ semana (total de quatro doses). Após as quatro primeiras semanas de tratamento, novos exames de imagem eram realizados para avaliar resposta de doença. $\mathrm{O}$ terceiro quimioterápico, doxorrubicina (50 $\mathrm{mg} / \mathrm{m}^{2} /$ dose), foi acrescentado nos casos de não redução tumoral ou progressão de doença. Todos os pacientes receberam no mínimo oito semanas de quimioterapia neoadjuvante. Um paciente recebeu carboplatina ao invés de dactinomicina em razáo da falta temporária desse quimioterápico no país.

A decisão quanto ao procedimento cirúrgico era tomada a partir da avaliação radiológica após as oito semanas de quimioterapia pré-operatória e realizada em conjunto pela equipe multidisciplinar. Cada rim foi tratado de forma individualizada, iniciando pelo rim menos acometido, seguido da abordagem do rim com doença mais avançada. O objetivo era realizar a abordagem cirúrgica mais conservadora possível para manter a melhor função renal e com critérios oncológicos (margens livres de doença).

A abordagem cirúrgica foi por incisão transversa supraumbilical ampla. Procedeu-se à inspeção de toda a cavidade abdominal à procura de lesóes associadas, não detectadas pelos exames pré-operatórios. $\mathrm{Na}$ sequência do isolamento do rim tumoral, o controle vascular era obtido por meio do isolamento da artéria e veia renal correspondente, e o leito cirúrgico foi mantido em 
hipotermia com gelo estéril. Foi demarcada no rim, com bisturi elétrico, uma linha limítrofe entre o tumor e a margem livre macroscópica antes da incisão cirúrgica no parênquima do rim abordado. A margem cirúrgica foi avaliada pelo patologista no estudo de congelação intraoperatório.

As complicaçôes potenciais avaliadas durante os procedimentos cirúrgicos foram: violação da cápsula tumoral, isquemia do parênquima remanescente por manipulação cirúrgica, extensão de trombos tumorais em grandes vasos, lesão extensa de cálices renais e de estruturas anatômicas adjacentes.

A quimioterapia pós-operatória foi baseada no estadiamento cirúrgico abdominal e na histologia, que foram classificados de acordo com o lado acometido pelo estádio mais avançado e mais agressivo, respectivamente. No pós-operatório, os pacientes receberam no mínimo 27 semanas de quimioterapia com dactinomicina e vincristina. A radioterapia foi indicada em casos selecionados, conforme as recomendaçóes do protocolo Siop 2001.

O seguimento desses pacientes foi feito com exames seriados de radiografia de tórax e ultrassonografia de abdome. A avaliação da função renal foi realizada por meio de exames laboratoriais e de cintilografia renal.

A sobrevida global foi calculada para dois e cinco anos neste estudo. O tempo de início da observação para cada indivíduo $\left(\mathrm{T}_{0}\right)$ foi definido como a data de matrícula no hospital especializado. A análise de sobrevivência considerou como evento a ocorrência de óbito por qualquer causa. Os pacientes que náo apresentaram o evento foram classificados como "censura" e contaram o tempo até a data da última observação feita em prontuário ou até a data final do estudo. As funçóes de sobrevida foram estimadas empregando-se o método de Kaplan-Meier. O teste log-rank foi aplicado para testar se as curvas diferiam entre categorias de uma mesma variável. A análise exploratória das variáveis e a análise de sobrevivência foram feitas no R, que é um ambiente de Programa livre para computaçẫo estatística e gráfica, utilizando os pacotes estatísticos Chron e Survival (R Core Team 2016) ${ }^{10}$.

O presente estudo foi aprovado pelo Comitê de Ética em Pesquisa (CEP) do INCA sob o ${ }^{\circ}$. CAAE 82799618.9.0000.5274.

\section{RESULTADOS}

As características dos 18 pacientes com tumor de Wilms bilateral sincrônico estão resumidas na Tabela 1. Dez pacientes eram do sexo feminino e oito do sexo masculino ( $\mathrm{F} / \mathrm{M}=1,25 / 1)$. A idade mediana ao diagnóstico foi 19 meses, variando de 3,4 a 63,1 meses.
Todos os pacientes apresentavam doença não metastática ao diagnóstico. O aumento do volume abdominal isolado ou associado à outra queixa clínica foi o sinal $/$ sintoma mais frequente, representando $77,8 \%$ $(\mathrm{n}=14)$ do total. Cinco $(29,4 \%)$ pacientes apresentaram anomalias constitucionais associadas: Denys-Drash $(\mathrm{n}=1)$, Beckwith-Wiedemann ( $\mathrm{n}=1)$, Frasier $(\mathrm{n}=1)$, hemi-hipertrofia ( $\mathrm{n}=1)$ e doença de Von Willebrand $(\mathrm{n}=1)$.

Todos os pacientes receberam quimioterapia pré-operatória com duas ou três drogas, com duração mediana de 15 semanas (variação de 7 a 29 semanas).

Os procedimentos cirúrgicos foram realizados em 17 pacientes, totalizando 32 unidades renais: $4 / 32$ biópsias (12,5\%); 8/32 CPTR (25\%); 3/32 nefrectomias parciais associadas à CPTR (9,4\%); 9/32 nefrectomias parciais $(28,1 \%)$ e $8 / 32$ nefrectomias totais (25\%) (Figura 1). Em 8/17 (47,1\%) pacientes, foi possível realizar CPTR, em uma ou duas unidades renais por paciente. Além disso, em 3/17 pacientes (17,6\%) com tumores multicêntricos, foi possível proceder à nefrectomia parcial combinada com CPTR no mesmo rim. No total, foi possível realizar cirurgia conservadora bilateral em $7 / 17$ pacientes $(41,2 \%)$. Dois pacientes não foram submetidos à cirurgia em um dos rins por apresentarem lesão residual puntiforme após a quimioterapia pré-operatória e, portanto, não tiveram registro histopatológico nesses rins. Uma criança com síndrome de Denys-Drash evoluiu para óbito por insuficiência renal grave durante a quimioterapia pré-operatória e também não teve estudo histopatológico.

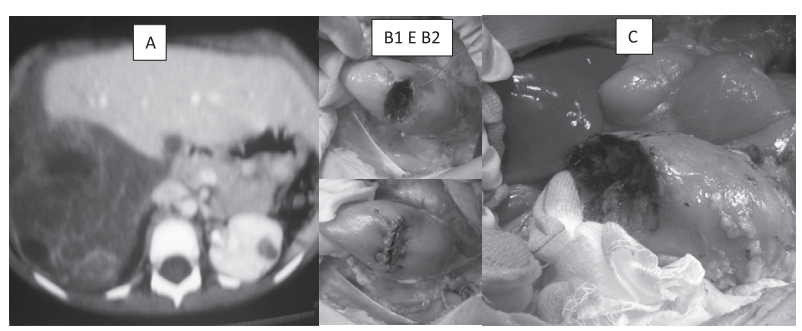

Figura 1. A figura 1A representa a tomografia computadorizada de abdome pré-operatória. As figuras 1B1 e 1B2 representam 0 procedimento CPTR no polo posterior do rim esquerdo. A figura $1 \mathrm{C}$ representa o procedimento CPTR no terço superior do rim direito

Nos 17 pacientes submetidos à cirurgia, os estádios cirúrgicos foram: sete, estádio I (39\%), cinco, estádio II $(27,8 \%)$, cinco, estádio III $(27,8 \%)$. Entre os pacientes com estádio cirúrgico III (ID 4, 6, 7, 9 e 10 na Tabela 1), um paciente apresentou limite cirúrgico comprometido por infiltraçáo neoplásica da cápsula renal e extravasamento ao tecido adiposo perirrenal, dois pacientes apresentaram ruptura tumoral e líquido peritoneal positivo para células neoplásicas, e os outros dois apresentaram implante em superfície peritoneal. 
Tabela 1. Características clínicas dos pacientes com tumor de Wilms bilateral, período 2000-2017

\begin{tabular}{|c|c|c|c|c|c|c|c|c|c|c|c|c|c|}
\hline \multirow{2}{*}{ ID } & \multirow{2}{*}{ Sexo } & \multirow{2}{*}{$\begin{array}{l}\text { Idade } \\
\text { (meses) }\end{array}$} & \multirow{2}{*}{$\begin{array}{l}\text { Malformação } \\
\text { associada }\end{array}$} & \multirow{2}{*}{$\begin{array}{l}\text { Quimioterapia } \\
\text { pré-operatória }\end{array}$} & \multicolumn{2}{|c|}{$\begin{array}{l}\text { Abordagens cirúrgicas } \\
\text { iniciais }\end{array}$} & \multicolumn{2}{|c|}{ Histologia } & \multirow{2}{*}{$\begin{array}{l}\text { Maior } \\
\text { estádio } \\
\text { cirúrgico }\end{array}$} & \multirow{2}{*}{ Recidiva } & \multirow{2}{*}{ Status } & \multirow{2}{*}{$\begin{array}{l}\text { Última } \\
\text { creatinina } \\
\text { (mg/dL) }\end{array}$} & \multirow{2}{*}{$\begin{array}{c}\text { Tempo de } \\
\text { seguimento } \\
\text { (em anos) }\end{array}$} \\
\hline & & & & & Rim direito & $\begin{array}{c}\text { Rim } \\
\text { esquerdo }\end{array}$ & Rim direito & $\begin{array}{c}\text { Rim } \\
\text { esquerdo }\end{array}$ & & & & & \\
\hline 1 & $\mathrm{~F}$ & 3,4 & & AV & CPTR & CPTR & TW HF e RN & TW HF e RN & I & & VSD & 0,8 & 14,4 \\
\hline 2 & M & 14,9 & $\begin{array}{l}\text { Beckwith- } \\
\text {-Wiedmann }\end{array}$ & AV & $\begin{array}{l}\text { Nefrectomia } \\
\text { total }\end{array}$ & CPTR & TW HF & TW HF e RN & 1 & $\begin{array}{l}\text { Pulmão e } \\
\text { rim }\end{array}$ & Óbito\& & 6,8 & 15,4 \\
\hline 3 & $\mathrm{~F}$ & 16,3 & & AV & Biópsia & $\begin{array}{c}\text { Nefrectomia } \\
\text { total }\end{array}$ & TW HF & TW HF e RN & I & & VSD & 0,6 & 16,6 \\
\hline 4 & M & 48,6 & & AV e AVD & $\begin{array}{l}\text { Nefrectomia } \\
\text { parcial }\end{array}$ & $\begin{array}{l}\text { Nefrectomia } \\
\text { parcial e } \\
\text { CPTR }\end{array}$ & TW HF (RI) & $\begin{array}{l}\text { TW HD (AR } \\
\text { AD) e RN }\end{array}$ & III & $\begin{array}{l}\text { Pulmão e } \\
\text { rim }\end{array}$ & Óbito & 0,4 & 2,0 \\
\hline 5 & $\mathrm{~F}$ & 33,5 & & AV e AVD & $\begin{array}{l}\text { Nefrectomia } \\
\text { total }\end{array}$ & sem cirurgia & $\begin{array}{l}\text { TW HF (RI } \\
\text { epitelial) }\end{array}$ & Desconhecido & 1 & & VSD & 0,6 & 14,1 \\
\hline 6 & M & 38,8 & $\begin{array}{c}\text { Hemi- } \\
\text {-hipertrofia }\end{array}$ & AV & Biópsia & $\begin{array}{c}\text { Nefrectomia } \\
\text { parcial }\end{array}$ & RN & $\begin{array}{c}\text { TW HF (RI tipo } \\
\text { misto) }\end{array}$ & III & Pulmão & VSD & 0,9 & 11,7 \\
\hline 7 & $\mathrm{~F}$ & 63,1 & & $A V$ e AVD & $\begin{array}{l}\text { Nefrectomia } \\
\text { parcial }\end{array}$ & CPTR & $\begin{array}{l}\text { TW HF (RI } \\
\text { estromal) }\end{array}$ & $\begin{array}{l}\text { TW HF (RI } \\
\text { epitelial e } \\
\text { estromal) }\end{array}$ & III & & VSD & 0,5 & 12,0 \\
\hline 8 & $\mathrm{~F}$ & 5,5 & Von Willebrand & $A V$ e AVD & Biópsia & $\begin{array}{c}\text { Nefrectomia } \\
\text { total }\end{array}$ & $\begin{array}{l}\text { TW HF (RI } \\
\text { estromal e } \\
\text { epitelial) }\end{array}$ & $\begin{array}{c}\text { TW HF (RI tipo } \\
\text { misto) }\end{array}$ & 1 & $\begin{array}{l}\text { Pulmão e } \\
\quad \text { rim }\end{array}$ & Óbito\& & 2,3 & 2,4 \\
\hline 9 & M & 49,7 & & AV & $\begin{array}{l}\text { Nefrectomia } \\
\text { parcial }\end{array}$ & $\begin{array}{c}\text { Nefrectomia } \\
\text { parcial }\end{array}$ & $\begin{array}{c}\text { TW HF (RI AF) } \\
\text { e RN }\end{array}$ & $\begin{array}{c}\text { TW HF (RI tipo } \\
\text { regressivo) }\end{array}$ & III & Fígado & VSD & 0,7 & 9,3 \\
\hline 10 & M & 61,1 & & $A V$ e AVD & $\begin{array}{l}\text { Nefrectomia } \\
\text { total }\end{array}$ & $\begin{array}{c}\text { Nefrectomia } \\
\text { parcial }\end{array}$ & $\begin{array}{c}\text { TW HF (RI tipo } \\
\text { misto) e RN }\end{array}$ & $\begin{array}{c}\text { TW HF (RI tipo } \\
\text { misto) e RN }\end{array}$ & III & Abdome & Óbito & 0,4 & 2,0 \\
\hline 11 & M & 11,2 & & $A V$ e AVD & CPTR & $\begin{array}{c}\text { Nefrectomia } \\
\text { parcial }\end{array}$ & RN & $\begin{array}{c}\text { TW HF (RI tipo } \\
\text { misto) }\end{array}$ & II & & VSD & 0,5 & 8,2 \\
\hline 12 & $F$ & 31,8 & & AV e AVD & $\begin{array}{l}\text { Nefrectomia } \\
\text { total }\end{array}$ & CPTR & $\begin{array}{c}\text { TW HD (AR } \\
\text { AD) }\end{array}$ & RN & I & Abdome & Óbito & 1,4 & 1,4 \\
\hline 13 & M & 5,0 & Denys-Drash & AV & Sem cirurgia & Sem cirurgia & Desconhecido & Desconhecido & Desconhecido & & Óbito* & 1,3 & $82^{*}$ \\
\hline 14 & M & 21,1 & Frasier & AV e AVD & $\begin{array}{l}\text { Nefrectomia } \\
\text { total }\end{array}$ & $\begin{array}{c}\text { Nefrectomia } \\
\text { parcial }\end{array}$ & $\begin{array}{c}\text { TW HF (RI } \\
\text { estromal) e RN }\end{array}$ & RN & II & & VSD & 0,5 & 6,0 \\
\hline 15 & $\mathrm{~F}$ & 11,9 & & AV & Biópsia & $\begin{array}{c}\text { Nefrectomia } \\
\text { parcial }\end{array}$ & RN & \begin{tabular}{|c|} 
TW HF (RI \\
epitelial) e RN \\
\end{tabular} & II & & VSD & 0,6 & 6,5 \\
\hline 16 & $F$ & 17,0 & & AV & $\begin{array}{l}\text { Nefrectomia } \\
\text { parcial e CPTR }\end{array}$ & CPTR & $\begin{array}{c}\text { TW HF (RI } \\
\text { epitelial) e RN }\end{array}$ & RN & 1 & & VSD & 0,3 & 5,1 \\
\hline 17 & $F$ & 6,3 & & AV e AVD & Sem cirurgia & $\begin{array}{c}\text { Nefrectomia } \\
\text { total }\end{array}$ & Desconhecido & $\begin{array}{c}\text { TW HF (RI tipo } \\
\text { misto) }\end{array}$ & II & Rim & VSD & 0,5 & 4,2 \\
\hline 18 & $\mathrm{~F}$ & 31,0 & & CDV & $\begin{array}{l}\text { Nefrectomia } \\
\text { parcial e CPTR }\end{array}$ & CPTR & $\begin{array}{c}\text { TW HD (AR } \\
\text { blastema) } \\
\text { e RN }\end{array}$ & $\begin{array}{l}\text { TW HF (RI } \\
\text { epitelial) }\end{array}$ & II & & VSD & 0,3 & 1,4 \\
\hline
\end{tabular}

Legendas: ID: paciente; CPTR: cirurgia preservadora de tecido renal; TW: tumor de Wilms; HF: histologia favorável; HD: histologia desfavorável; RN: resto nefrogênico; $\mathrm{AD}$ anaplasia difusa; $\mathrm{AF}$ : anaplasia focal; RI: risco intermediário, $\mathrm{AR}$ : alto risco; $\mathrm{AV}$ : actinomicinaD e vincristina; $\mathrm{AVD}$ : actinomicinaD, vincristina e doxorrubicina; CDV: carboplatina, doxorrubicina e vincristina; VSD: vivo sem doença; $82^{*}$ : 82 dias; óbito*: por toxicidade; óbito\&: anéfrico.

Em relação à histologia, restos nefrogênicos estavam presentes em $13 / 17$ dos pacientes (76,5\%), histologia favorável em $14 / 17$ pacientes $(82,4 \%)$ e histologia desfavorável em $3 / 17$ pacientes (17,6\%).

As complicaçôes evidenciadas durante a primeira etapa cirúrgica de cada paciente foram: ruptura de cápsula tumoral $(\mathrm{n}=2)$, isquemia do parênquima remanescente por manipulação cirúrgica $(n=1)$ e lesão de veia renal $(n=1)$.

Durante o acompanhamento, oito pacientes $(47,1 \%)$ apresentaram recidiva de doença: pulmão e $\operatorname{rim}(n=3)$, abdome ( $\mathrm{n}=2)$, fígado $(\mathrm{n}=1)$, pulmáo $(\mathrm{n}=1)$ e rim $(\mathrm{n}=1)$. Cinco desses pacientes (ID 2, 4, 8, 10 e 12) evoluíram para óbito por progressão de doença e os demais (ID 6, 9 e 17) permanecem vivos sem evidência de doença.
Após a recidiva, dois (11\%) pacientes tornaram-se anéfricos: paciente ID 2 apresentava síndrome de Beckwith-Wiedmman. Foi submetido à nefrectomia total à direita e biópsia de pequena lesão em rim esquerdo. A histologia evidenciou tumor de Wilms de histologia favorável e restos nefrogênicos. $\mathrm{O}$ paciente permaneceu em controle durante 12 anos e, após esse período, apresentou recidiva em rim esquerdo com anaplasia difusa. Foi tratado com vários esquemas de quimioterapia e radioterapia abdominal, sem sucesso. Houve necessidade de ressecção do rim remanescente e evoluiu para óbito por progressão de doença 2,6 anos após a recidiva; paciente ID 8 apresentava doença de Von Willebrand. Foi submetida à nefrectomia total à esquerda e à biópsia de pequena lesão em terço 
médio de rim direito. $\mathrm{O}$ diagnóstico anatomopatológico mostrou tumor de Wilms de histologia favorável em ambos os rins. Apresentou recidiva em rim direito, nove meses após a primeira cirurgia. Recebeu vários esquemas de quimioterapia e radioterapia abdominal, inclusive com necessidade de nefrectomia total direita. Evoluiu para óbito em progressão de doença 1,3 anos após a recidiva (Tabela 1).

Ocorreram seis óbitos no total: cinco por progressão de doença e um por toxicidade (paciente com síndrome de Denys-Drash).

Todos os pacientes que sobreviveram apresentavam funçâo renal normal na última avaliação. $\mathrm{O}$ tempo mediano de seguimento para a coorte do estudo foi de 6,3 anos $(0,22$ a 16,6 anos $)$ e a sobrevida global em dois e cinco anos para todos os 18 pacientes estudados, incluindo histologia favorável e desfavorável, foi de $82,6 \%$, e 70,8\%, respectivamente (Gráfico 1). Para pacientes com histologia favorável, a sobrevida global em dois e cinco anos foi de $92,9 \%$ e 85,7\%, respectivamente, e apresentou significância estatística (p-valor $<0,0001$ ). Por outro lado, para pacientes com histologia desfavorável, a sobrevida global em dois anos foi de 50\%. A sobrevida global em cinco anos, segundo o tipo cirurgia realizada, foi de $87,5 \%$ para CPTR e/ou nefrectomia parcial (cirurgia conservadora) e de 62,8\% nefrectomia total $(\mathrm{p}=0,0001)$ (Gráfico 2).

\section{DISCUSSÃO}

Em consonância com a literatura, o presente estudo retrospectivo encontrou $7 \%$ de pacientes com tumor de Wilms bilateral sincrônico no período em análise $e^{4}$. A sobrevida global em cinco anos para todos os 18 pacientes com tumor de Wilms bilateral sincrônico foi de 70,8\%.

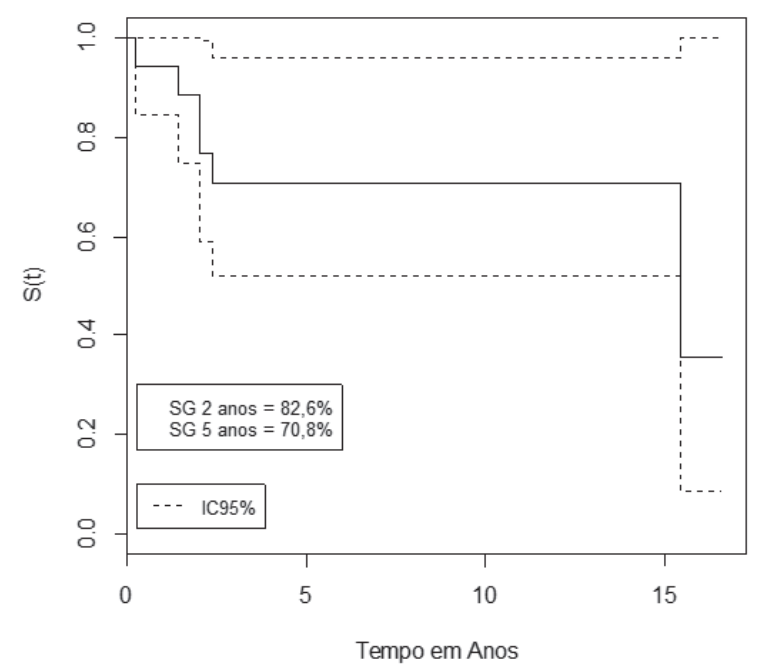

Gráfico 1. Sobrevida global em dois e cinco anos para tumor de Wilms bilateral sincrônico ( $n=18), 2000-2017$

Legendas: IC: intervalo de confiança; SG: sobrevida global.

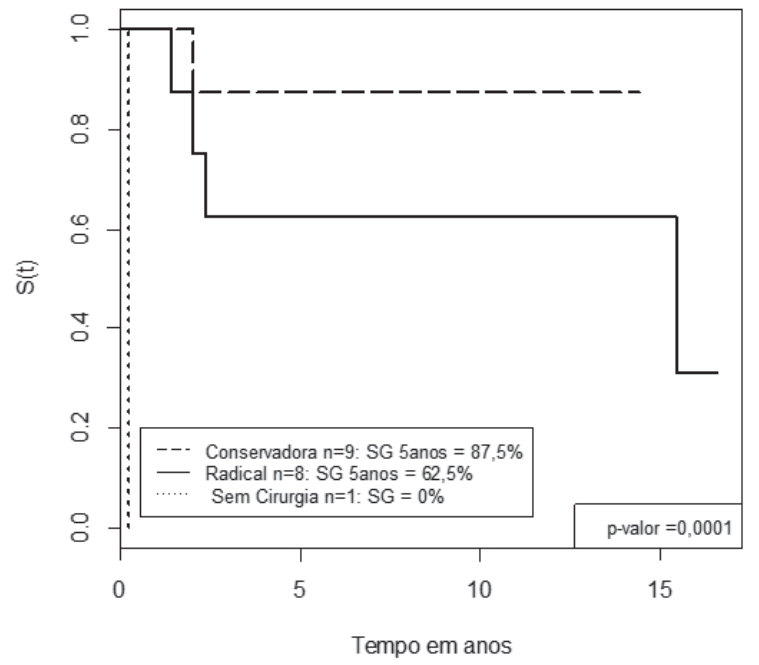

Gráfico 2. Sobrevida Global em cinco anos para tumor de Wilms bilateral sincrônico, segundo o tipo de cirurgia, 2000-2017

Legenda: SG: sobrevida global.

Segundo o tipo de cirurgia, a sobrevida global em cinco anos foi de $87,5 \%$ para os pacientes submetidos à CPTR e à nefrectomia parcial. Esses índices são semelhantes aos estudos do NWTS-5 e da Associazione Italiana di Ematologia Oncologia Pediatrica (AIEOP), que relatam sobrevida global em quatro anos para todos os pacientes com tumor de Wilms bilateral sincrônico de $80,8 \%{ }^{11}$ e de $80 \%{ }^{12}$, respectivamente, porém inferiores à sobrevida global relatada pelo Children Oncology Group (COG) de $94,16 \%{ }^{11}$.

$\mathrm{O}$ tumor de Wilms bilateral tende a se manifestar mais em lactentes ${ }^{7}$. Na presente pesquisa, a idade mediana ao diagnóstico foi de 19 meses, acometendo crianças mais jovens do que as referidas na literatura, que variam de 24 meses $^{12}$ a 30 meses de idade ${ }^{4}$. Restos nefrogênicos estavam presentes em $76,5 \%$ dos pacientes, semelhante ao descrito na literatura ${ }^{8}$. O tumor de Wilms bilateral sincrônico é mais comumente associado a síndromes genéticas que frequentemente se correlacionam com doença renal intrínseca. Podem ser multifocais e apresentam maior risco para desenvolver falência renal, em consequência de cirurgia agressiva, sendo a causa mais comum a remoção cirúrgica dos néfrons e em alguns casos nefrectomia bilateral ${ }^{6-8}$. No presente estudo, duas crianças foram submetidas à nefrectomia bilateral em consequência de doença em progressão, semelhante ao encontrado na literatura mundial ${ }^{5}$.

Na tentativa de evitar a falência renal, o papel da CPTR para crianças com o tumor de Wilms bilateral sincrônico tornou-se cada vez mais importante, embora a CPTR bilateral ainda não seja amplamente realizada. Davidoff et al. ${ }^{6}$ relatam que foi viável realizar com segurança CPTR bilateral em 92,9\% (39/42) pacientes com tumor de 
Wilms bilateral sincrônico, alcançando bom resultado oncológico. Segundo os autores, o uso de quimioterapia pré-operatória com três drogas (AVD) nessa série pode ter contribuído para a maior facilidade em realizar CPTR.

No entanto, o estudo conduzido pela AIEOP em 90 pacientes com tumor de Wilms bilateral sincrônico não encontrou maior proporção de CPTR bilateral nos 37 (32\%) pacientes que receberam quimioterapia pré-operatória AVD, quando comparado ao grupo de 43 $(58 \%)$ de pacientes que receberam duas drogas $(\mathrm{AV})^{12}$. Além disso, o estudo prospectivo multi-institucional para tratamento de crianças com Tumor de Wilms bilateral coordenado pelo grupo COG alcançou 39\% de CPTR bilateral em 242 pacientes avaliados ${ }^{11}$.

O presente estudo realizou CPTR em 47,1\% dos pacientes e a combinação de CPTR com nefrectomia parcial em um mesmo rim em $17,6 \%$ dos pacientes, alcançando bom resultado oncológico e de função renal. No total, foi possível a realização de cirurgia conservadora bilateral em $41,2 \%$ (7/17) dos pacientes. Na nossa série, diferentemente do relatado por Davidoff et al. ${ }^{6}$, a quimioterapia pré-operatória era iniciada com AV e a doxorrubicina era acrescentada somente em casos de não redução tumoral após as quatro primeiras semanas de tratamento. Isso talvez tenha contribuído para o menor percentual de CPTR bilateral.

Mesmo em casos de tumor de Wilms bilateral sincrônico com envolvimento de hilo renal é possível realizar CPTR com segurança, utilizando a técnica de nefrectomia parcial longitudinal descrita por Fuchs ${ }^{13}$. O sucesso cirúrgico é essencial para o resultado favorável nesses pacientes. Para tanto, é fundamental que sejam tratados em centros de referência com oncologistas e cirurgióes pediátricos com grande expertise, conforme preconizado pelo protocolo Siop 2001.

Outro aspecto importante é o tempo de duração da quimioterapia pré-operatória; isto é, o tempo entre o início da quimioterapia e a primeira ressecção cirúrgica. A quimioterapia pré-operatória é utilizada visando à redução tumoral e a facilitar a cirurgia conservadora. No presente estudo, a quimioterapia pré-operatória teve duração mediana de 15 semanas. Diversos autores reportam que estender o tempo de quimioterapia neoadjuvante para além de três meses (12 semanas) não aumentou a proporção de CPTR bilateral. Além disso, as pesquisas relatam que o máximo de redução tumoral ocorreu nas primeiras 12 semanas de quimioterapia pré-operatória. Ademais, a falha na resposta à quimioterapia neoadjuvante pressupóe duas condiçôes histológicas: diferenciação em tecido estromal maduro ou transformação anaplásica ${ }^{11,12,14}$.

Uma das limitaçóes deste estudo foi de ele ter sido realizado de forma retrospectiva, podendo acarretar viés de seleção e informação.
Pacientes com tumor de Wilms bilateral sincrônico precisam ser acompanhados indefinidamente para avaliar complicaçóes relacionadas ao tratamento, a possibilidade de recorrência tumoral e a monitoração da função renal a longo prazo ${ }^{15}$.

\section{CONCLUSÃO}

O tratamento do tumor de Wilms bilateral sincrônico é complexo e requer ação conjunta multidisciplinar visando ao melhor resultado oncológico. A CPTR em crianças com o tumor de Wilms bilateral sincrônico é viável e pode ser realizada com segurança em centros de referência. A preservação cirúrgica do parênquima renal é fundamental para o controle da doença e a conservação da função renal nessas crianças que estão em risco significativo de insuficiência renal.

\section{CONTRIBUIÇÕES}

Todos os autores participaram igualmente da pesquisa, coleta de dados, interpretação dos resultados, análise crítica do conteúdo, no desenvolvimento do manuscrito bem como na análise e revisão do artigo na sua parte final para envio do manuscrito para publicação.

\section{AGRADECIMENTOS}

À equipe da Oncologia Pediátrica, da Cirurgia Pediátrica, ao arquivo médico e à Secretaria da Pediatria do HCI/INCA/RJ.

\section{DECLARAÇÃO DE CONFLITO DE INTERESSES}

Nada a declarar.

\section{FONTES DE FINANCIAMENTO}

Não há.

\section{REFERÊNCIAS}

1. Instituto Nacional de Câncer José Alencar Gomes da Silva. Incidência, mortalidade e morbidade hospitalar por câncer em crianças, adolescentes e adultos jovens no Brasil: informaçóes dos registros de câncer e do sistema de mortalidade. Rio de Janeiro: INCA, 2016.

2. Doganis D, Panagopoulou P, Tragiannidis A, Georgakis MK, Moschovi M, Polychronopoulou S, et al. Childhood nephroblastoma in Southern and Eastern Europe and the US: incidence variations and temporal trends by human development index. Cancer Epidemiol. 2018;54:75-81.

3. Chu A, Heck JE, Ribeiro KB, Brennan P, Boffetta P, Buffler P, et al. Wilms' tumour: a systematic review of risk 
factors and meta-analysis. Paediatr Perinat Epidemiol. 2010;24(5):449-469.

4. Ehrlich PF. Bilateral Wilms tumor: the need to improve outcomes. Expert Rev Anticancer Ther. 2009;9(7):963-973.

5. Giel DW, Williams MA, Jones DP, Davidoff AM, Dome JS. Renal function outcomes in patients treated with nephron sparing surgery for bilateral Wilms tumor. J Urology 2007;118(4):1786-1790.

6. Davidoff AM, Interiano RB, Wynn L, Delo Santos N, Dome JS, Green DM, et al. Overall survival and renal function of patients with Synchronous Bilateral Wilms Tumor undergoing surgery at a single institution. Ann. Surg. 2015;262(4):570-576.

7. Kieran K, Williams MA, McGregor LM, Dome JS, Krasin MJ, Davidoff AM. Repeat nephron-sparing surgery for children with bilateral Wilms tumor. J Pediatr Surg. 2014;49(1):149-153.

8. Kieran K, Davidoff AM. Nephron-sparing surgery for bilateral Wilms tumor. Pediatr Surg Int. 2015;31(3):229-236.

9. McKenna AM, Keating LE, Vigneux A, Stevens S, Williams A, Geary DF. Quality of life in children with chronic kidney disease-patient and caregiver assessments. Nephrol Dial Transplant. 2006;21(7):1899-1905.

10. R Foundation for Statistical Computing. The R Project for Statistical Computing. [computer program]. R version 3.3.2. [Internet]. [place unknown]: The $\mathrm{R}$ foundation; 2016. [cited 2016 Out 31]. Available from: https://www.R-project.org.

11. Ehrlich P, Chi YY, Chintagumpala MM, Hoffer FA, Perlman EJ, Kalapurakal JA, et al. Results of the first prospective multi-institutional treatment study in children with bilateral Wilms tumor (AREN0534): a report from the children's oncology group. Ann Surg. 2017;266(3):470-478.

12. Indolfi P, Jenkner A, Terenziani M, Crocoli A, Serra A, Collini P, et al. Synchronous bilateral Wilms tumor: a report from the Associazione Italiana Ematologia Oncologia Pediatrica (AIEOP). Cancer. 2013;119(8):1586-1592.

13. Fuchs J, Szavay P, Seitz G, Handgretinger R, Schäfer JF, Warmann SW. Nephron sparing surgery for synchronous bilateral nephroblastoma involving the renal hilus. J Urol. 2011;186(4):1430-36.

14. Shamberger RC, Haase GM, Argani P, Perlman EJ, Cotton CA, Takashima J, et al. Bilateral Wilms' tumors with progressive or nonresponsive disease. J Pediatr Surg. 2006;41(4):652- 657.

15. Sarhan OM, El-Baz M, Sarhan MM, Ghali AM, Ghoneim MA. Bilateral Wilms' tumor: single-center experience with 22 cases and literature review. Urology 2010;76(4):946-951 